\title{
CETÁCEOS FÓSILES (MAMMALIA, ODONTOCETI, EURHINODELPHINOIDEA, INIOIDEA, PHYSETERIOIDEA) DE LA FORMACIÓN CURRÉ, MIOCENO SUPERIOR (HEMPHILLIANO TEMPRANO TARDÍO) DE COSTA RICA
}

\author{
FOSSIL CETACEA (MAMMALIA, ODONTOCETI, EURHINODELPHINOIDEA, \\ INIOIDEA, PHYSETERIOIDEA) OF THE CURRÉ FORMATION, UPPER \\ MIOCENE (EARLY-LATE HEMPHILLIAN) OF COSTA RICA
}

\author{
Ana L. Valerio ${ }^{1} \&$ César A. Laurito ${ }^{1,2 *}$ \\ ${ }^{1}$ Departamento de Historia Natural, Museo Nacional de Costa Rica. Apdo. \\ 749-1000, San José \\ ${ }^{2}$ Instituto Nacional de Aprendizaje \\ *Autor para contacto: cesarlaurito@ice.co.cr
}

(Recibido: 29/08/2011 ; aceptado: 11/06/2012)

\begin{abstract}
Four new records of Odontoceti are described for the Upper Miocene of Southern Central America; these founds suggest a strength sea connection between the North Atlantic Coast of Europe, the Coastal Plain of North America with the tropical East Pacific Ocean. On the other hand, the fossil record of the river dolphin Goniodelphis sp., shows an important affinity between the ancient coastal wetlands of Florida and the epicontinental sediments of the Curré Formation at Costa Rica.

Key words: Eurhinodelphinidae, Iniidea, Kentriodontidae, Physeteridae, Upper Miocene, Costa Rica.

RESUMEN: Cuatro nuevos registros de Odontoceti son descritos para el Mioceno Superior del sur de América Central; estos hallazgos sugieren una fuerte conexión marina entre la costa del Atlántico Norte de Europa, la Planicie Costera de América del Norte con el trópico del Pacífico oriental. Por otro lado el registro fósil del delfín de río Goniodelphis sp., muestra una importante afinidad entre los antiguos humedales costeros de la Florida y los sedimentos epicontinentales de la Formación Curré en Costa Rica.

Palabras clave: Eurhinodelphinidae, Iniidae, Kentriodontidae, Physeteridae, Mioceno Superior, Costa Rica.
\end{abstract}




\section{INTRODUCCIÓN}

Los cetáceos son mamíferos marinos que comparten un ancestro común con los Artiodactyla (Uhen, 2010), como predadores acuáticos desarrollaron extraordinarias adaptaciones evolutivas tanto a nivel fisiológico como morfológico, diferenciándose notablemente de sus predecesores terrestres o incluso de otros mamíferos marinos (Simpson, 1945; Gingerich, 2005).

Los Neoceti o cetáceos actuales, constituyen un grupo monofilético derivado de los Basilosauridae (Geisler \& Sanders, 2003; Uhen, 2004, 2010), este a su vez se divide en dos grupos monofiléticos (Geisler \& Sanders, 2003) de acuerdo a sus mecanismos de alimentación, a saber, las ballenas o Mysticeti y los cetáceos dentados u Odontoceti; el registro fósil de este último grupo puede seguirse hasta el Oligoceno Tardío o incluso cerca del límite Eoceno/Oligoceno (Barnes \& Goedert, 2000) y está compuesto por marsopas, delfines y ballenas de esperma.

El registro de cetáceos fósiles para Costa Rica era prácticamente inexistente hasta que recientemente Laurito et al. (2011) dieron a conocer el hallazgo del odontoceto Squalodon especie indeterminada, proveniente del Mioceno Medio de la Formación Río Banano, provincia de Limón. Previo a ello, Valerio (2010) y Laurito \& Valerio (2010) habían mencionado, de manera general, la aparición de restos de cetáceos en la Formación Curré pero estos no habían sido estudiados aún.

La localidad fosilífera de San Gerardo de Limoncito, está ubicada en el cantón de Coto Brus, distrito 4to Limoncito, provincia de Puntarenas, Costa Rica; en las coordenadas geográficas 8051'19.6"N/8304'51.9"W (Fig. 1).

Esta localidad ha producido un variado registro de peces, reptiles y mamíferos terrestres, cuyos restos se asocian a sedimentos de origen fluvial y abanicos deltaicos subacuáticos, que se alternan con sedimentos infralitorales depositados en un antiguo y extenso estuario. Estas facies sedimentarias se corresponden estratigráficamente con el techo de la Formación Curré de edad Mioceno Medio a Superior y la fauna de vertebrados, en particular la de caballos, permite establecer una edad mamífero norteamericana Hemphilliano temprano - tardío (Valerio, 2010; Laurito \& Valerio, 2010).

La fauna de mamíferos terrestre se caracteriza por la mezcla de elementos de abolengo suramericano (básicamente xenarthras) y norteamericano (caballos, gonfotéridos, camélidos y tayasuidos), lo que la distingue como la fauna centroamericana con la evidencia más antigua y contundente de intercambio entre las Américas, vía América Central meridional.

Entre los vertebrados de hábitos anfibios y acuáticos, destaca la asociación de crocodílidos, bagres y tortugas, en especial trionychidos que sugieren un ambiente hipohalino a dulceacuícola y entre los vertebrados marinos, una modesta fauna de tiburones y rayas que reafirma la proximidad del ambiente marino eurihalino.

El registro de mamíferos marinos se limitaba a unas cuantas vértebras que confirmaban la presencia de probables cetáceos mysticetos; sin embargo, el registro de odontocetos no había sido confirmado. Una reciente revisión de la colección de la fauna de San Gerardo de Limoncito, permitió la identificación de varios elementos dentales correspondientes a cuatro diferentes géneros de odontocetos, objeto de estudio del presente escrito. Este material se encuentra depositado en la Colección de Fósiles, de la Sección de Geología del Departamento de Historia Natural del Museo Nacional de Costa Rica.

\section{PALEONTOLOGÍA}

La clasificación utilizada en la presente publicación está fundamentada en Barnes, 1985; Ichishima et al., 1994; Fordyce \& Barnes, 1994; Dawson, 1996; Lambert, 2005. 


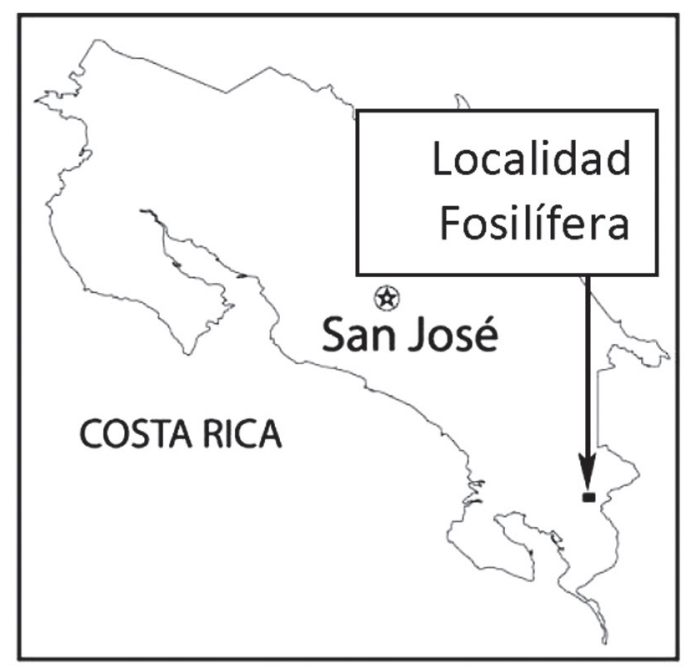

Fig. 1: Mapa de ubicación.

Orden Cetacea Brisson, 1762

Suborden Odontoceti Flower, 1867

Infraorden Eurhinodelphinida Fordyce \& de Muizon, 2001

Superfamilia Eurhinodelphinoidea, de Muizon 1988

Familia Eurhinodelphinidae Abel, 1901

Género Eurhinodelphis Du Bus, 1867

\section{Eurhinodelphis sp.}

Material: el ejemplar CFM-1868 corresponde a un diente aislado de $20,89 \mathrm{~mm}$, de probable posición anterior (figuras 2a-a").

Descripción: diente de corona cónica de 8,57 $\mathrm{mm}$ de altura, con crenulaciones aisladas, presenta una muesca que abarca toda la altura de la corona en su cara comisural. La raíz es de forma rectangular, ligeramente sigmoidea, comprimida antero-posteriormente y es notablemente más grande que la corona.

Discusión: diente cuya forma y tamaño general recuerda a los dientes anteriores de los géneros Kentriodon y Delphinodon, pero la corona carece

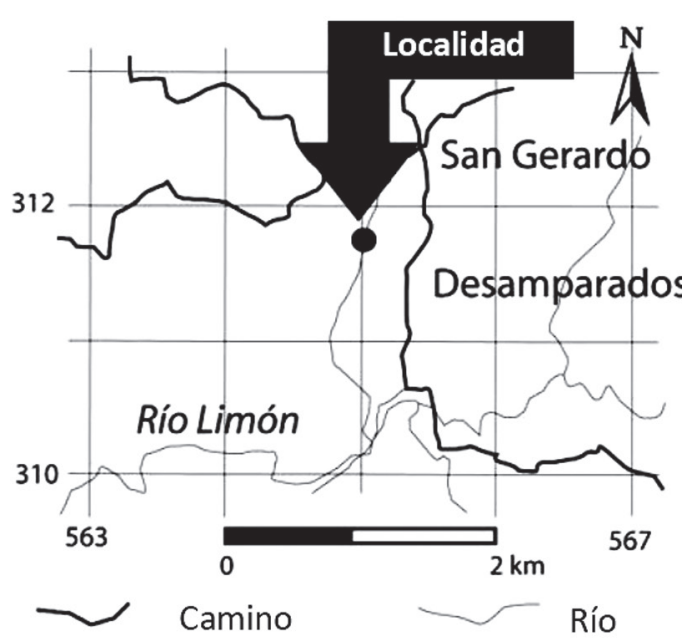

de las denticulaciones accesorias propias de dichos géneros, lo que facilita su determinación dentro del género Eurhinodelphis. Aunque se acepta la existencia de dos especies fósiles, Eurhinodelphis cocheteuxi Du Bus, 1867 y Eurhinodelphis longirostris Du Bus, 1867, un solo elemento dental no es suficiente para asignarlo a una de ellas.

Distribución estratigráfica: en el Viejo Mundo el género Eurhinodelphis se ha registrado en el Mioceno de Holanda, Langhiano - Serravalliano de Bélgica (Misonne, 1958; Lambert, 2005), Tortoniano de Italia (Pilleri, 1986); Mioceno Superior tardío de Turquía (Piveteau, 1978) y en el Mioceno Temprano Medio, Langhiano de Japón (Oichi \& Hasegawa, 1995).

En América del Norte se ha registrado en el Burdigaliano - Langhiano de Maryland y Virginia (Kellogg, 1925, 1965a).

Infraorden Delphinida de Muizon, 1984

Superfamilia Inioidea de Muizon, 1984

Familia Iniidae (Gray, 1846)

Género Goniodelphis Allen, 1941 


\section{Goniodelphis sp.}

Material: el ejemplar CFM-1866 corresponde a 1 diente aislado de posición incierta con la corona fragmentada (figuras $2 \mathrm{c}-\mathrm{c} ")$.

Descripción: diente de $26,30 \mathrm{~mm}$ de altura, con corona cónica rota, ligeramente volcada hacia la comisura y en sentido lingual en particular la porción superior; en el esmalte se observan estriaciones longitudinales. La raíz es lobulada en forma de delta, conspicuamente expandida en su extremo distal, ligeramente inflada en su mitad superior y comprimida en sentido vestíbulolingual; presenta un cuello en su porción superior que marca el límite basal de la corona.

Discusión: El diente CFM-1866 presenta una amplia raíz comprimida linguo-vestibularmente que recuerda tanto a los delfines Iniidae como a los delfines marinos de la familia Ziphiidae. Además, presenta un característico cuello en la base de la corona que permite diferenciarle fácilmente de esta última familia y relacionarlo con el género Goniodelphis. Otra característica importante de este género, es la forma marcadamente triangular de la raíz que en el ejemplar del presente estudio se muestra redondeado, quizás producto de la dilución gástrica o por erosión, debido al transporte por el agua. Estas dos características son descritas por Allen (1941) y Kellogg (1944).

Distribución estratigráfica: la especie Goniodelphis hudsoni Allen, 1941 se ha descrito para el Serravalliano-Hemphilliano tardío de Bone Valley Formation en Polk County, Florida, Estados Unidos (Morgan, 1994).

Superfamilia Physeteroidea Gray, 1868

Familia Physeteridae Gray, 1821

Subfamilia Physeterinae Gray, 1821

Género Orycterocetus Leidy, 1853

\section{Orycterocetus sp.}

Material: el ejemplar CFM-1870 corresponde a un fragmento apical de la corona de 24,96 $\mathrm{mm}$ (figuras 2b-b').

Descripción: fragmento apical de la corona de contorno cilíndrico, ligeramente curvo con ex- tremo romo y estrías longitudinales. Se observa, además, una amplia cavidad pulpar.

Discusión: los dientes del género Orycterocetus son cilíndricos, delgados, relativamente largos y ligeramente curvos. Presentan morfologías variadas y en algunos dientes se observan ápices redondeados con canales o estrías longitudinales. El fragmento apical descrito en el presente trabajo cumple con estas características y es muy similar a los ilustrados por Kellogg (1965b, Lámina 30, Fig. 13) y por Bianucci et al. (2004, Fig. 2(5)).

Distribución paleobiogeográfica y estratigráfica: el género Orycterocetus se ha registrado en el Viejo Mundo en el Burdigaliano tardío Messiniano temprano del sur de Italia (Bianucci et al., 2004) y un diente aislado es descrito para el Burdigaliano de Faluns de la Loire en el Norte de Francia (Ginsburg \& Janvier, 1975). En el Langhiano - Burdigaliano de la Formación Berchem en el área de Antwerp, Bélgica (Lambert, 2008; Louwye et al., 2010).

En América del Norte el género Orycterocetus se ha registrado en el Mioceno de Maryland y Virginia en la Formación Calvert (Kellogg, 1965b)

Superfamilia Delphinoidea Flower, 1865

Familia Kentriodontidae Slijper, 1936

Subfamilia Lophocetinae Barnes, 1978

Genus Hadrodelphis Kellogg, 1966

\section{Hadrodelphis sp.}

Material: el ejemplar CFM-3294 corresponde a un diente inferior, posterior, probablemente izquierdo, de 27,29 mm (figuras 2d-d"').

Descripción: diente de corona subcónica de $9,63 \mathrm{~mm}$, parcialmente rota, cuya mitad inferior es bulbosa y la superior ligeramente volcada en sentido vestíbulo-lingual, con crenulaciones aisladas tanto en el ápice como en la base. La raíz es protuberante, ésta se amplía desde un cuello comprimido en la base de la corona, hasta alcanzar una forma bulbosa en la parte media para luego terminar aplanándose y comprimiéndose en su mitad inferior. 


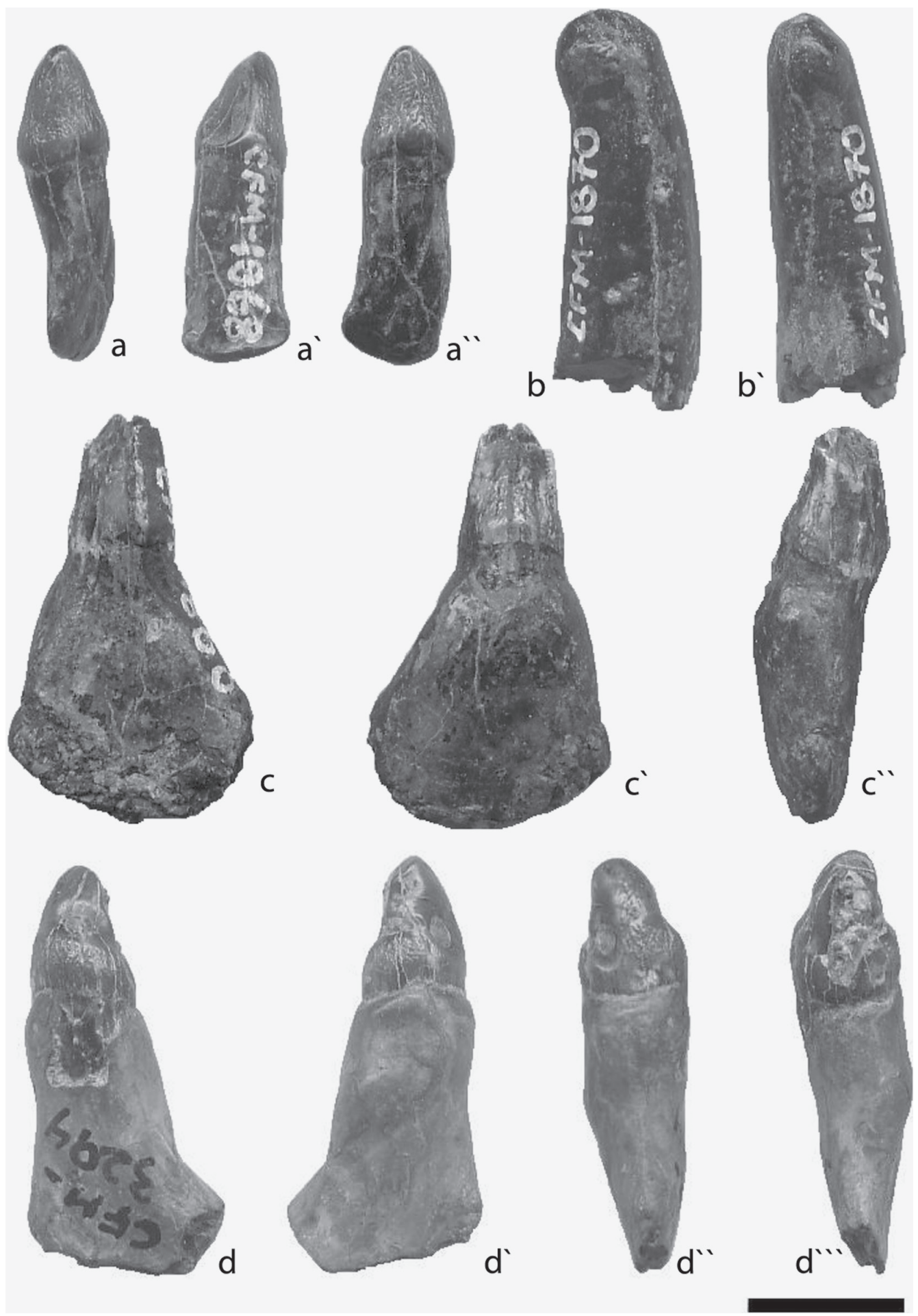

Fig. 2: Eurhinodelphis sp., en vistas: a. lateral, a'. posterior, a".anterior; Orycterocetus sp. , b. lateral, b'. lingual; Goniodelphis sp., c. lingual, c'. labial, c". lateral; Hadrodelphis sp., d. labial, d'. lingual, d". anterior, d"”. posterior. Escala visual $1 \mathrm{~cm}$. 
Discusión: diente de forma y talla similar a los de la especie norteamericana Hadrodelphis calvertensis Kellogg, 1966 (Lámina 45, Figs. 1-3) y Dawson (1996, Fig. 6); notablemente más grande que los dientes de la especie europea Hadrodelphis poseidon Ginsburg \& Janvier, 1971. A pesar de ello, no es posible establecer la pertenencia a una especie determinada, pues no se cuenta con otros elementos óseos que permitan una mejor diferenciación y determinación del material.

De hecho la especie Hadrodelphis poseidon Ginsburg \& Janvier, 1971 se fundamenta en dos dientes aislados por lo que su validez taxonómica a nivel de especie es cuestionada por Dawson (1996).

Distribución paleobiogeográfica y estratigráfica: el género Hadrodelphis y la especie $H$. poseidon se ha documentado en el Helvetiano de las localidades de Noyant-sul-le-Lude y Pontigné en el Departamento de Maine-et-Loire, provincia D’Anjou, en Francia (Ginsburg \& Janvier, 1975).

En América del Norte la especie $H$. calvertensis se ha registrado en el Mioceno Medio de la Formación Calvert en Calvert County e Indian Creek en Charles County, ambos en el Estado de Maryland (Kellogg, 1966; Dawson, 1996) y en el Mioceno de Florida en las localidades de Gainsville en Alachua County; en Florida central y en Gadsden County (Morgan, 1994; Hulbert \& Morgan, 2001).

\section{Paleobiogeografía de los delfines de la Formación Curré}

Los hallazgos costarricenses de Hadrodelphis sp. y Goniodelphis sp. suponen una fuerte vinculación de primer orden entre el sur de América Central y la provincia Caribeña con la península de Florida. Este tipo de correlaciones paleoprovinciales se ha dado con otros elementos faunísticos, por ejemplo los tiburones de la Formación Uscari (Laurito, 1999).

En el caso del delfín Hadrodelphis sp., su género se caracterizó por una distribución ambiatlántica norte-hemisférica, durante el Mioceno
Medio y Superior. En Europa, sus registros se restringen al Atlántico de la mitad norte de Francia y en América del Norte su distribución se concentra en las Formaciones Calvert en Maryland y Bone Valley de Florida, ambas del Mioceno, confirmando así, una distribución paleogeográfica a lo largo de la provincia de la Planicie Costero Atlántica al norte y la provincia Floridana al sur. Está vinculación paleobiogeográfica también es confirmada por el registro ambiatlántico y norte-hemisférico del género Orycterocetus en el Mioceno Medio de Bélgica y el norte de Francia, y los registros de la Formación Calvert en los estados de Maryland y Virginia en Estados Unidos.

Tanto Hadrodelphis como Orycterocetus sugieren una vinculación paleobiogeográfica de segundo orden entre la Formación Curré, en el Pacífico de América Central meridional, con las faunas miocenas atlánticas al norte de la península de Florida y la costa de Francia y Bélgica. Esta no es la primera evidencia de este tipo de distribución en el Mioceno Medio y Superior; Pyenson \& Hoch (2007) consideran que la distribución paleogeográfica de otra familia de delfines, los Pontoporiidae que en la actualidad viven exclusivamente en las aguas marino someras de América del Sur y en la cuenca del río Ganges en Asia, alcanzaron el Mar del Norte de previo al Mioceno Tardío vía Mar de Tethys, pero luego cuando el enfriamiento climático se incrementó, esta fauna de delfines abandonó el Mar del Norte y el Atlántico Norte. Uno de sus géneros Pontistes Burmeister, 1885; se ha hallado en el Mioceno Tardío de la formaciones Paraná en Argentina (Cozzuol, 1985), Pisco en Perú (de Muizon \& DeVries, 1985) y el suroeste de Dinamarca (Pyenson \& Hoch, 2007); su distribución solo se explica debido a una dispersión vía el corredor marino de América Central que conectó las aguas del Pacífico oriental y el Atlántico Norte hasta que se completó el cierre del istmo de Panamá entre 5.0 y $3.5 \mathrm{Ma}$ (Keigwin, 1982; Duque-Caro, 1990). Teniendo lo anterior en cuenta, una dispersión en sentido contrario, explicaría el hallazgo de Hadrodelphis y Orycterocetus en el Pacífico del sur de América Central, misma que se pudo haber dado de previo al Mioceno Tardío. 
En el caso específico del delfín de agua dulce, Goniodelphis sp., se evidencia una conexión y distribución epicontinental entre la Florida y el Pacífico sur de Costa Rica cuya paleobiogeografía solo se explicaría por algún grado de tolerancia al agua marina eurihalina de las costas caribeñas de América Central y del estrecho oceánico al sur del istmo de Panamá, ello a pesar de que los delfines ribereños actuales de acuerdo con Hamilton et al. (2001), se restringen a ecosistemas dulceacuícolas.

Un patrón similar de distribución epicontinental al de Goniodelphis sp., se observa al comparar la paleobiogeografía de las tortugas dulceacuícolas de la familia Trionychidae, muy comunes en los sedimentos terciarios de América del Norte (Meylan, 1987). Sus restos se han registrado en el Mioceno del norte de Venezuela (Wood \& Patterson, 1973; Sánchez-Villagra et al., 2004), en el extremo meridional del Caribe y también asociados a los odontocetos de la Formación Curré (Laurito et al., 2005). Tal patrón de distribución durante el Mioceno solo se puede explicar, asumiendo algún tipo de tolerancia a los ambientes marinos y a la existencia de grandes humedales asociados a desembocaduras de antiguos ríos que se extendían a lo largo de la costa, facilitando la migración de estos organismos dulceacuícolas (Laurito et al., 2005).

En suma, la fauna de delfines de la Formación Curré se caracteriza por un conjunto de cetáceos típicos del Mioceno tardío que confirman una vinculación de tercer grado con las faunas marinas mediterráneas del Mioceno Medio y Superior, una vinculación de segundo grado con las faunas del Atlántico norte o ambiatlánticas y una vinculación de primer orden con las faunas de la Florida. Si se tiene en cuenta la posible distribución pantropical del Eurhinodelphis para el Mioceno tardío, se destaca la conexión Tethysiano-Mediterránea con el Pacífico de América Central meridional.

\section{REFERENCIAS BIBLIOGRÁFICAS}

ALLEN, G.M., 1941: A fossil river dolphin from Florida.- Bull. Mus. of Comparative Zool. 89(1):1-8.
BARNES, L.G., 1985: The Late Miocene dolphin Pithanodelphis Abel, 1905 (Cetacea: Kentriodontidae) from California.Contributions in Sci., Nat. Hist. Mus. of Los Angeles County, 367:1-27.

BARNES, L.G., \& GOEDERT, J.L., 2000: The world's oldest known odontocete (Mammalia, Cetacea).- J. Vertebrate Paleont., Abstracts of Papers 20 (supplement 3): 28.

BIANUCCI, G., LANDINI, W. \& VAROLA, A., 2004: First discovery of the Miocene northern Atlantic sperm whale Orycterocetus in the Mediterranean.- Geobios, 37(5): 569-573.

COZZUOL, M.A., 1985: The Odontoceti of the 'Mesopotamiense' of the Parana River Ravines. Systematic review.- En: PILLERI, G. (ed.): Investigations on Cetacea.- Brain Anat. Inst., Univ. of Berne, 17: 39-54.

DAWSON, S., 1996: A description of the skull and postcrania of Hadrodelphis calvertense Kellogg 1966, and its position within the Kentriodontidae (Cetacea; Delphinoidea).J. Vertebrate Paleont. 16(1): 125-134.

DUQUE-CARO, H., 1990: Neogene stratigraphy, paleoceanography and paleobiogeography in northwest South America and the evolution of the Panama Seaway.- Palaeogeography, Palaeoclimat. and Palaeoecol. 77: 203 234.

FLOWER, W.H., 1867: Description of the skeleton of Inia geoffrensis.- Trans. Zool. Soc. Lond. 6: 87-116.

FORDYCE, R.E. \& BARNES, L.G., 1994: The evolutionary history of whales and dolphins.- Annu. Rev. Earth Planet. Sci. 22: 419-455. 
GEISLER, J.H. \& SANDERS, A.E., 2003: Morphological evidence for the phylogeny of Cetacea.- J. Mamm. Evol. 10: 23-129.

GINGERICH, P.D., 2005: Cetacea. - En: ROSE, K.D. \& ARCHIBALD, J.D. (eds.): Placental mammals: origin, timing, and relationships of the major extant clades.Johns Hopkins Univ. Press, Baltimore: 234-252.

GINSBURG, L. \& JANVIER, P., 1971: Les mammifères marins des faluns miocènes de la Touraine et de l'Anjou.- Bull. Mus. Nat. d'Hist. Nat., Sci. de la Terre, 6(22): 161195.

GINSBURG, L. \& JANVIER, P., 1975: Les mammifères marins des faluns de la Touraine et de l'Anjou: faune, gisements et paléobiologie.- Bull. Soc. E. Sci. Anjou, N. S. 9: 73-96.

HAMILTON, H., CABALLERO, S., COLLINS, A.G. \& BROWNELL, R.L., 2001: Evolution of river dolphins.- Proc. R. Soc. Lond. Biol. Sci. 268: 549-556.

HULBERT, R.C. \& MORGAN, G.S., 2001: Mammalia 9: Whales and dolphins. En: HULBERT, R.C. (ed.): The Fossil Vertebrates of Florida.- Univ. Press of Florida, Gainesville: 331-342.

ICHISHIMA, H., BARNES, L., FORDYCE, E., KIMURA, A. \& BOHASKA, D., 1994: A review of Kentriodontine dolphins (Cetacea; Delphinoidea; Kentriodontidae): Systematics and Biogeography.- The Island Arc, 3: 486-492.

KEIGWIN, L.D., Jr., 1982: Isotopic paleoceanography of the Caribbean and East Pacific: role of Panama uplift in late Neogene time.- Sci. 217: 350-353.
KELLOGG, R., 1925: On the occurrence of remains of fossil porpoises of the genus Eurhinodelphis in North America.- Proc. of the United States Nat. Mus. 66(26): $1-40$.

KELLOGG, R., 1944: Fossil cetaceans from the Florida Tertiary.- Bull. Mus. Comparative Zool. at Harvard College, 94(9): 433471.

KELLOGG, R., 1965a: A new whalebone whale from the Miocene Calvert Formation.- Bull. United States Nat. Mus. 247(1): 1-45.

KELLOGG, R., 1965b: The Miocene Calvert sperm whale Orycterocetus. - Bulletin of the United States National Museum, 247(2):47-63.

KELLOGG, R., 1966: A new odontocete from the Calvert Miocene of Maryland.Bull.United States National Mus. 247(4): 99-101.

LAMBERT, O., 2005: Phylogenetic affinities of the long-snouted dolphin Eurhinodelphis (Cetacea, Odontoceti) from the Miocene of Antwerp, Belgium.- Palaeontol. 48(3): 653-679.

LAMBERT, O., 2008: Sperm whales from the Miocene of the North Sea: a re-appraisal.- En: STEURBAUT, E., JAGT, J.W. \& JAGT-YAZYKOVA, E.A. (eds.): Annie V. Dhondt Memorial Volume.- Bull. de l'Institut Royal des Sci. Nat. de Belgique, Sciences de la Terre, 78: 277-316.

LAURITO, C., 1999: Los seláceos fósiles de la localidad de Alto Guayacán (y otros ictiolitos asociados), Mioceno Superior-Plioceno Inferior de la Formación Uscari, provincia de Limón, Costa Rica.- 168 págs. LAURITO, C. (ed.), San José. 
LAURITO, C. \& VALERIO, A., 2010: Los caballos fósiles de la Formación Curré, cantón de Coto Brus, Costa Rica.- 131 págs. Mus. Nac. de Costa Rica, San José.

LAURITO, C.A., VALERIO, A.L., HERNANDEZ, A.C. \& OVARES, E., 2011: Primer registro de un cetáceo fósil (Mammalia, Cetacea, Odontoceti, Squalodontidae) en la Formación Río Banano, Mioceno Medio de Costa Rica, América Central.- Rev. Geol. Amér. Central, 44: 153-156.

LAURITO, C., VALERIO, A.L., GOMEZ, L.D., MEAD, J.I., PEREZ, E. \& PEREZ, L.G., 2005: A Trionychidae (Reptilia: Testudines, Cryptodira) from the Pliocene of Costa Rica, southern Central America.Rev. Geol. Amér. Central, 32:7-11.

LOUWYE, S., MARQUET, R., BOSSELAERS, M. \& LAMBERT, O., 2010: Stratigraphy of an early-middle Miocene sequence near Antwerp in northern Belgium (southern north sea basin).- Geologica Belgica, 13(3): 269-284.

MEYLAN, P.A., 1987: The phylogenetic relationships of soft-shelled turtles (family Trionychidae).- Bull. Amer. Mus. Nat. Hist. 186: 1-110.

MISONNE, X., 1958: Faune du Tertiare et $\mathrm{du}$ Pléistocène inférieur de Belgique (Oiseaux et mammifères).- Bull. de l'Institut Royal des Sci. Nat. de Belgique, 34(5): 1-36.

MORGAN, G.S., 1994: Miocene and Pliocene marine mammal faunas from the Bone Valley Formation of central Florida.Contributions in Marine Mammal Paleont., Proceedings of the San Diego Soc. Nat. Hist. 29: 239-268.
DE MUIZON, C. \& DEVRIES, T.J., 1985: Geology and paleontology of late Cenozoic marine deposits in the Sacaco area (Peru).Geologische Rundschau, 74: 547-563.

OISHI, M. \& HASEGAWA, Y., 1995: A list of fossil cetaceans in Japan.- The Island Arc, 3: 493-505.

PILLERI, G., 1986: The Miocene Cetacea of the Pietra Leccese with special reference to the Cosimo de Giorgi Collection, Lecce.- 27 págs. Univ of Berne, Suiza.

PIVETEAU, M.J., 1978: Un nouveau gisement de Vertèbres dans le Chersonian KurtchukTchenkmedje Ouest (Thrace turque).Comptes Rendus Hebdomadaires des séances de L'Académie des Sciences, Series D 287(5): 455-458.

PYENSON, N.D. \& HOCH, E., 2007: Tortonian pontoporiid odontocetes from the Eastern North Sea.- J. Vertebrate Paleont. 27(3): 757-762.

SÁNCHEZ-VILLAGRA, M.R., ASHER, R.J., RINCÓN, A.D., CARLINI, A., MEYLAN, P. \& PURDY, W., 2004: New faunal reports for the cerro La Cruz locality (Lower Miocene), North-Western Venezuela.- En: SÁNCHEZ-VILLAGRA, M.R. \& CLACK, J.A. (eds): Fossil of the Miocene Castillo Formation, Venezuela: Contributions on Neotropic Palaentology.Spec. Papers in Paleont. 71: 105-112.

SIMPSON, G.G., 1945: The principles of classification and a classification of mammals.- Bull. Amer. Mus. Nat. Hist. $85: 1-350$.

UHEN, M.D., 2004: Form, function, and anatomy of Dorudon atrox (Mammalia, Cetacea): an Archaeocete from the Middle to Late Eocene 
of Egypt.- Univ. Mich. Mus. Paleontol. Pap. Paleontol. 34: 1-222.

UHEN, M.D., 2010: The origin(s) of Whales.Annu. Rev. Earth Planet. Sci. 38:189-219.

VALERIO, A., 2010: Paleontología, bioestratigrafía y paleoecología de los caballos fó- siles de la Formación Curré en el Cantón de Coto Brus, Costa Rica (análisis basado en material dental).- 353 págs. Univ. de Costa Rica, San José [Tesis Lic.].

WOOD, R.C. \& PATTERSON, B., 1973: A fossil Trionychid turtle from South America.-Breviora, 405:1-10. 